\title{
Cross-clamping of the descending thoracic aorta leads to the asymmetrical distribution of propofol during cardiopulmonary bypass surgery
}

\author{
Maiko Yamauchi-Satomoto ${ }^{1}$, Yushi U. Adachi ${ }^{2}$, Tadayoshi Kurita ${ }^{3}$, Koji Morita $^{4}$, and Shigehito Sato ${ }^{2,3}$ \\ ${ }^{1}$ Department of Critical Care Medicine, Tokyo Medical and Dental University, Tokyo, ${ }^{2}$ Intensive Care Unit of University Hospital, \\ ${ }^{3}$ Department of Anesthesiology and Resuscitation, ${ }^{4}$ Operating Division, Hamamatsu University School of Medicine, Shizuoka, Japan
}

Background: We hypothesized that cross-clamping of the descending thoracic aorta (CcDTA) would result in significant changes in plasma propofol concentrations $(\mathrm{Cp})$ proximal and distal to the cross-clamp. We investigated the effect of CcDTA on Cp centrally and distally, including the pulmonary artery and the cardiopulmonary bypass (CPB) cannula.

Methods: The bispectral index (BIS) was recorded during CcDTA in eight patients undergoing thoracic aortic surgery using target-controlled total intravenous anesthesia with propofol. The calculated $\mathrm{Cp}$ was maintained at $3 \mu \mathrm{g} / \mathrm{ml}$. Cp was measured in blood samples drawn from the right radial artery, left dorsalis pedis artery, pulmonary artery, and the long venous CPB cannula.

Results: Complete data were obtained from six patients. BIS decreased significantly in all cases 5 minutes after initiating CcDTA. BIS continued to decrease in association with increasing propofol concentrations. During CcDTA, $\mathrm{Cp}$ in samples from the radial and pulmonary arteries ( $3.5 \pm 0.50$ and $2.9 \pm 0.63 \mu \mathrm{g} / \mathrm{ml}$, mean $\pm \mathrm{SD}$ ) was significantly higher than in samples from the dorsalis pedis artery and the venous cannula $(1.1 \pm 0.22$ and $1.4 \pm 0.02 \mu \mathrm{g} / \mathrm{ml})(\mathrm{P}<$ 0.05).

Conclusions: The results suggest that almost all of the blood returning from the superior vena cava during CcDTA directly enters the pulmonary circulation without mixing with blood from the inferior vena cava. Observed changes in anesthetic blood concentrations could be due to the presence of a split circulation and asymmetrical distribution of propofol induced by CcDTA and CPB. (Korean J Anesthesiol 2012; 62: 327-331)

Key Words: Bispectral index (BIS) monitor, Cardiopulmonary bypass, Propofol.

Received: February 24, 2011. Revised: 1st, July 21, 2011; 2nd, August 17, 2011. Accepted: August 28, 2011.

Corresponding author: MaikoYamauchi-Satomoto, M.D., Ph.D., Department of Critical Care Medicine, Tokyo Medical and Dental University, 1-5-45 Yushima, Bunkyo-ku, Tokyo 113-8519, Japan. Tel: 81-3-5803-4718, Fax: 81-3-5803-5652, E-mail: satomoto.maiko8@gmail.com (c) This is an open-access article distributed under the terms of the Creative Commons Attribution Non-Commercial License (http:// creativecommons.org/licenses/by-nc/3.0/), which permits unrestricted non-commercial use, distribution, and reproduction in any medium, provided the original work is properly cited. 


\section{Introduction}

Total intravenous anesthesia has become a popular clinical technique over the last decade $[1,2]$. We recently encountered a case showing a dubious decrease in BIS values to $<10$ during cross-clamping of the descending thoracic aorta (CcDTA). Kakinohana et al. [3] reported that the blood concentration of propofol (Cp) increases immediately after CcDTA during thoracic aortic aneurysm repair surgery, whereas BIS values decrease. The BIS value of one of their patients decreased to zero under propofol anesthesia during CcDTA [4]. Cp values from the radial artery were approximately two to three times higher than those from the femoral artery. However, Kakinohana et al. [4] could not exclude the possibility that decreased hepatic clearance of propofol was responsible for the difference induced by CcDTA. It is possible that the pharmacokinetics of some drugs administered during CcDTA might be altered due to changes in the distribution, metabolism, and excretion of the drugs [5-7]. Thus, differences in blood concentrations above and below the cross clamp would be expected.

In this investigation, we simultaneously measured $\mathrm{Cp}$ values in blood samples taken from the right radial artery, left dorsalis pedis artery, pulmonary artery, and a long venous CPB cannula. The aim of the study was to determine whether or not split circulation occurs during CcDTA.

\section{Materials and Methods}

After obtaining institutional approval from the Institutional Review Board and the Ethics Committee of Hamamatsu University School of Medicine, Hamamatsu, Shizuoka, Japan, eight patients (American Society of Anesthesiologists physical status II or III; age, 36-80 yr) were enrolled. The patients were scheduled for elective thoracic or thoracoabdominal aneurysm repair surgery requiring partial extracorporeal cardiopulmonary by-pass (CPB). Written informed consent was obtained from all patients participating in this study.

After establishing routine monitors and bispectral index (BIS) monitoring (Aspect A-1050; Aspect Medical Systems, Natick, MA, USA), anesthesia was induced by the target-control infusion (TCI) technique using a TCI pump (TE-371; Terumo, Tokyo, Japan) with $3 \mu \mathrm{g} / \mathrm{ml}$ of propofol into venous access of the right arm. The blood concentration of fentanyl resulting from intermittent administration was predicted using a personal digital assistant (Visor Edge; Handspring Japan, Tokyo, Japan) and software (Palmacokinetics Ver 0.98; Osamu Uchida, http:// homepage1.nifty.com/o-uchida/palmacokinetics/). The calculated blood concentration of fentanyl was maintained at 2-4 $\mathrm{ng} / \mathrm{ml}$ during anesthesia. Neuromuscular blockade was achieved with $0.1 \mathrm{mg} / \mathrm{kg}$ vecuronium. The trachea was intubated with a double-lumen endotracheal tube, and all patients were mechanically ventilated to achieve an end-tidal carbon dioxide concentration of 30-35 mmHg. Anesthesia was maintained with propofol TCI and intermittent injections of fentanyl and vecuronium via an upper limb vein. BIS values were maintained at 40-60 prior to CcDTA.

Before the surgery, the right radial and left dorsalis pedis arteries were cannulated, and the pulmonary artery was catheterized. Cardiac output was monitored using the continuous thermodilution technique (OptiQ; Hospira Japan, Osaka, Japan). Patients were placed in the right decubitus position with the lower half of the body supinated anteriorly to facilitate right femoral vessel cannulation. After isolating both the right femoral artery and vein, an arterial cannula was inserted and then attached to the CPB pump oxygenator. A long venous CPB cannula (21 Fr $50 \mathrm{~cm}$, Bio-Medicus Cannula; Medtronic, Minneapolis, MN, USA) was introduced into the right femoral vein, and the catheter tip was advanced to a position between the inferior vena cava and the right atrium. The tip was confirmed to be correctly positioned by transesophageal echocardiography (NEMIO ssA-550A; Toshiba, Tokyo, Japan). Thereafter, the operation commenced.

The distal clamp site was slightly different for different patients. All patients required one-lung ventilation. Clamp range was from distal to the left subclavian artery to proximal to the celiac trunk.

Body temperatures in the rectum and bladder were kept at $35.1^{\circ} \mathrm{C}$ and $35.3^{\circ} \mathrm{C}$, respectively, during partial CPB. Throughout the investigation period, Cp was kept at $3 \mu \mathrm{g} / \mathrm{ml}$ using TCI. Blood samples to measure $\mathrm{Cp}$ values were drawn from the arterial lines (right radial artery, left dorsalis pedis artery, and pulmonary artery) and the venous line (long venous CPB cannula) before CcDTA; at 5, 10, 20, and 40 min after starting CcDTA; and at 0, 5, 10, and 20 min after releasing the CcDTA. BIS values were recorded at the same time as blood samples were collected. Plasma was separated by centrifugation and stored at $-40^{\circ} \mathrm{C}$. Propofol concentration was determined by high-performance liquid chromatography (LC-10 series; Shimadzu, Kyoto, Japan) with fluorescence detection at $310 \mathrm{~nm}$ after excitation at $276 \mathrm{~nm}$ (RF550; Shimadzu, Kyoto, Japan) [8].

Data are presented as means \pm SDs. The BIS and Cp values were analyzed using a repeated-measures analysis of variance (ANOVA). The Neumann-Keuls post hoc test was applied to assess differences between before and during CcDTA or after releasing CcDTA. An ANOVA was used to compare Cp in blood simultaneously sampled from the radial, dorsalis pedis, and pulmonary arteries, as well as the venous cannula. Statistical significance was defined as $\mathrm{P}<0.05$. 


\section{Results}

Data from six patients were evaluated (Table 1). Two patients were excluded because a steady and appropriate depth of anesthesia, as estimated by the BIS value, could not be achieved during the operation. Mean aortic cross clamping time was 54.7 $\pm 17.6 \mathrm{~min}$. The default perfusion flow rate was about $1.2 \mathrm{~L} /$ $\mathrm{min} / \mathrm{m}^{2}$ during partial $\mathrm{CPB}$, and the mean pressure of the distal aorta monitored from femoral arterial pressure was maintained at $>60 \mathrm{mmHg}$. When the pressure decreased to $<60 \mathrm{mmHg}$, the CPB flow rate was increased appropriately. Cardiac output, measured by continuous thermodilution using a pulmonary artery catheter, decreased significantly from $4.5 \pm 1.3 \mathrm{~L} / \mathrm{min} / \mathrm{m}$ 2 to $3.8 \pm 0.9 \mathrm{~L} / \mathrm{min} / \mathrm{m}^{2}$ during CcDTA and returned to $5.0 \pm 1.7$ $\mathrm{L} / \mathrm{min} / \mathrm{m}^{2}$ after CcDTA.

BIS values were $42-63$ before CcDTA and continued to decrease at the same time as $\mathrm{Cp}$ in the upper limb increased (Fig. 1). BIS was significantly lower than the baseline value 20 minutes after starting CcDTA $(\mathrm{P}<0.05)$.

No significant difference was found between the $\mathrm{Cp}$ values sampled from the radial, dorsalis pedis, and pulmonary arteries before CcDTA. However, the Cp values of the radial artery and the pulmonary artery gradually increased during CcDTA, and levels in both arteries were significantly higher than preclamping values 40 minutes after commencing CcDTA $(\mathrm{P}<$ 0.05) (Fig. 2). In contrast, $\mathrm{Cp}$ values of the venous $\mathrm{CPB}$ cannula and the dorsalis pedis artery were lower during CcDTA. The Cp values in the pulmonary artery were approximately two to three times higher than those in the long venous $\mathrm{CPB}$ cannula.

No differences in $\mathrm{Cp}$ values were observed among all sampling locations after release of CcDTA, and BIS values recovered to pre-CcDTA levels. None of the patients showed any neurological deficits during the postoperative period, including cerebral infarction or paraplegia resulting from spinal cord ischemia.

\section{Discussion}

The results of this study clearly demonstrate an asymmetrical distribution of propofol during CcDTA. This phenomenon can be explained by the presence of a split circulation induced by CcDTA and CPB. Changes in BIS values were reflected in changes in the Cp of the upper body.

Kakinohana et al. [3] reported that Cp increased, whereas BIS value decreased, immediately after starting CcDTA during propofol anesthesia. Using step-by-step methods throughout

Table 1. Demographic and Surgery-related Data of the Six Patients

\begin{tabular}{|c|c|c|c|c|c|c|c|}
\hline Patient ID & Age (yr) & Sex & Body weight (kg) & Operation time (min) & Anesthesia time (min) & CPB time (min) & ASA physical state \\
\hline 1 & 35 & Male & 74 & 222 & 366 & 31 & 2 \\
\hline 2 & 80 & Male & 56 & 227 & 365 & 56 & 2 \\
\hline 3 & 76 & Male & 50 & 198 & 375 & 45 & 3 \\
\hline 4 & 66 & Male & 68 & 399 & 533 & 84 & 2 \\
\hline 5 & 66 & Female & 48 & 245 & 388 & 60 & 2 \\
\hline 6 & 75 & Male & 51 & 267 & 394 & 52 & 2 \\
\hline
\end{tabular}

CPB: cardiopulmonary bypass, ASA: American Society of Anesthesiologists.
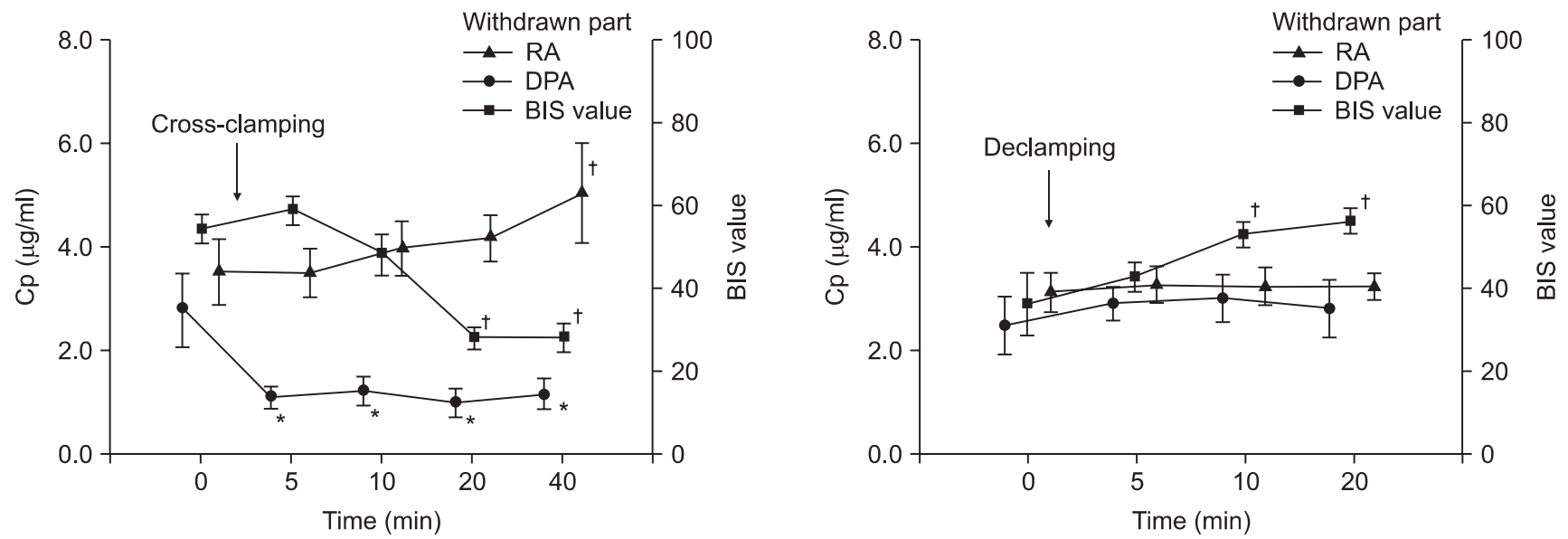

Fig. 1. The effects of cross clamping and declamping of the descending thoracic aorta on BIS values and plasma concentrations of propofol. Cp: plasma propofol concentration, RA: radial artery, DPA: dorsalis pedis artery, BIS: bispectral index. $* \mathrm{P}<0.05$ compared to $\mathrm{Cp}$ in the RA. ${ }^{\dagger} \mathrm{P}<0.05$ compared to values before cross-clamping. 

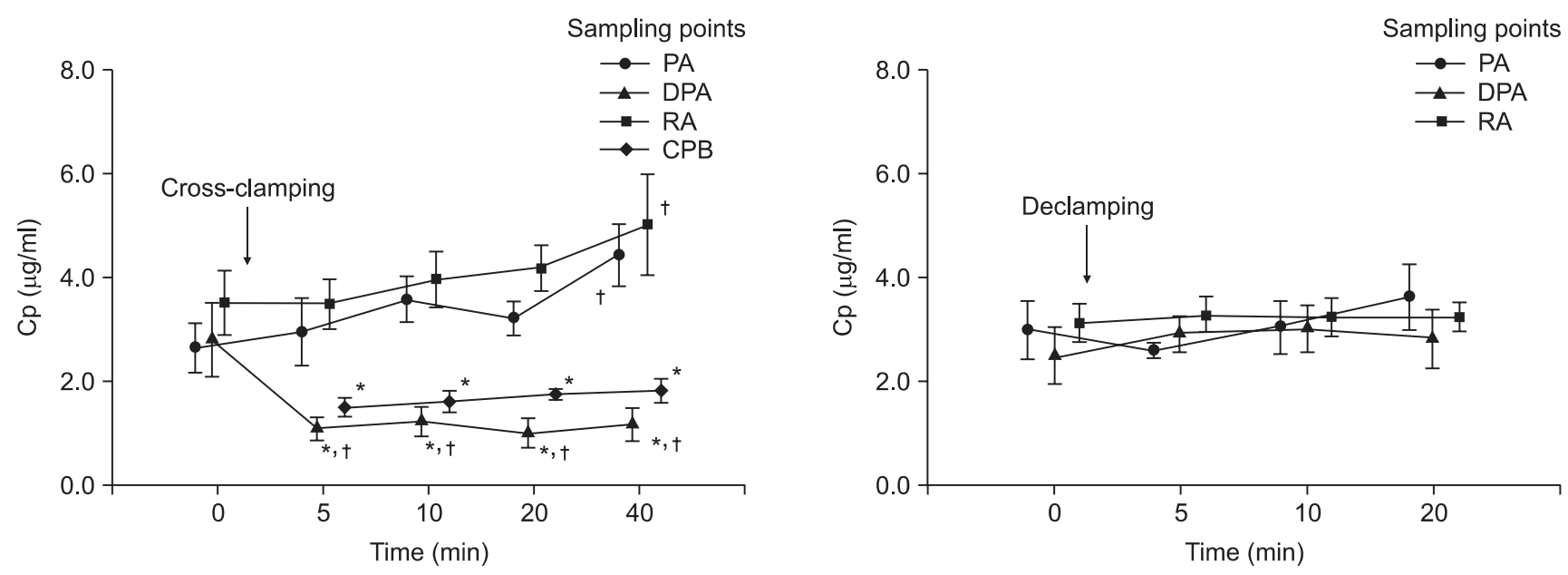

Fig. 2. The effects of cross clamping and declamping of the descending thoracic aorta on plasma propofol concentration. Cp: plasma propofol concentration, PA: pulmonary artery, DPA: dorsalis pedis artery, RA: radial artery, CPB: cardiopulmonary bypass inlet. ${ }^{*} \mathrm{P}<005$ compared to the $\mathrm{Cp}$ in the $\mathrm{PA} .{ }^{\dagger} \mathrm{P}<0.05$ compared to the values before cross-clamping.

CcDTA (as a precaution against BIS values decreasing to $<30$ ), significant differences in the $\mathrm{Cp}$ values between radial and femoral artery blood samples were also detected. There are two possible mechanisms for these phenomena [3]. First, CcDTA may change circulatory conditions and reduce circulatory volume. This change could reduce the distribution volume of propofol relative to total body weight that is infused into an upper limb vein and increase $\mathrm{Cp}$ in the proximal body [1].

The second possible mechanism is reduced hepatic clearance of propofol during CcDTA. Abdominal organs including the liver are mainly perfused through the celiac, supra mesenteric, and inferior mesenteric arteries. The output during CPB might be smaller than that of normal circulation, resulting in a reduction in regional blood flow. A previous investigation [3] could not exclude this explanation for the significant regional difference in $\mathrm{Cp}$. We found that $\mathrm{Cp}$ values decreased in the long venous CPB cannula during CcDTA in comparison to the radial and pulmonary arteries before CcDTA. If decreased hepatic clearance was the only cause for the differing $\mathrm{Cp}$ values, $\mathrm{Cp}$ in the CPB cannula should have increased during CcDTA. We conclude that the high propofol concentrations in the radial and pulmonary arteries were not due to reduced hepatic clearance.

$\mathrm{Cp}$ in the pulmonary artery differed from $\mathrm{Cp}$ in the dorsalis pedis artery and the CPB cannula. Cp values in the pulmonary artery were approximately two to three times higher than those in the long CPB cannula. Thus, significant differences in Cp were observed between the pulmonary artery and the long CPB cannula, which might suggest that almost all of the blood returning from the superior vena cava entered the pulmonary circulation directly during CcDTA, almost without mixing with blood from the inferior vena cava. These results suggest that we demonstrated the presence of a split circulation and asymmetrical distribution of propofol induced by CcDTA and CPB.

BIS values decreased during CcDTA, and these results support the hypothesis that increased Cp enhanced anesthesia. However, hypothermia during CPB has also been reported to decrease BIS values [9]; thus, the interpretation of the BIS values in the current study might require further attention. The body temperatures of the patients were kept at $35.1^{\circ} \mathrm{C}$ and $35.3^{\circ} \mathrm{C}$ during partial CPB, and this mild hypothermia may have caused the decreased BIS values.

A long CPB cannula was introduced into the right femoral vein, and the catheter tip was advanced to a position between the inferior vena cava and the right atrium. This cannula has two inlets at the tip; one around the right atrium and one around the inferior vena cava. Correct positioning of the tip was confirmed by transesophageal echocardiography, and the correct position should have facilitated withdrawal of mixed venous blood. However, the final position of the cannula was determined by cardiovascular surgeons and the CPB operator. Sufficient blood volume was their biggest concern during the procedure, and optimal positioning for withdrawal of mixed venous blood might not have provided adequate blood volume for withdrawal.

No significant differences were found among the Cp samples during surgical declamping of the aorta. Immediately before the surgical declamping procedure, the CPB flow was reduced and physiological forward perfusion began with partial release of CcDTA. Split perfusion may have ceased during the preparation for complete declamping, and, hence, no differences in Cp were observed. The disappearance of the split circulation will require further intensive investigation.

We studied only a small number of patients, but the changes 
observed were consistent statistically significant and were found in all six patients. Furthermore, one patient was under CPB for only 31 minutes. At first, approval was obtained for 16 patients to participate in the study. However, after collecting data from six patients, we recognized that the propofol concentration was clearly different between the upper body and lower body during cross-clamping of the descending thoracic aorta. So, we stopped collecting data and changed anesthesia from $3 \mu \mathrm{g} / \mathrm{ml}$ TCI propofol to BIS-dependent TCI propofol, although the six patients had no neurological complications due to decreased BIS values.

In summary, our results confirmed a difference in $\mathrm{Cp}$ between the pulmonary artery and venous $\mathrm{CPB}$ cannula during CcDTA. The results indicate that almost all of the blood returning from the superior vena cava entered the pulmonary circulation directly, without mixing with blood from the inferior vena cava. Anesthesiologists should be conscious of the potential for a split circulation and asymmetric distribution of propofol during CcDTA with CPB and adjust dosing accordingly.

\section{References}

1. Billard V, Gambus PL, Chamoun N, Stanski DR, Shafer SL. A comparison apectral edge, delta power and bispectral index as EEG measures of alfentanil, propofol, and midazolam drug effect. Clin Pharmacol Ther 1997; 61: 45-58.
2. Milne SE, Kenny GN. Future application for TCI systems. Anaesthesia 1998; 53 Suppl 1: 56-60.

3. Kakinohana M, Nakamura S, Fuchigami T, Miyata Y, Sugahara $\mathrm{K}$. Influence of the descending thoracic aorta cross clamping on Bispectral Index value and plasma propofol concentration in humans. Anesthesiology 2006; 104: 939-43.

4. Kakinohana M, Miyata Y, Kawabata T, Kawashima S, Tokumine J, Sugahara K. Bispectral Index decreased to "0" in propofol anesthesia after a cross-clamping of descending thoracic aorta. Anesthesiology 2003; 99: 1223-5.

5. Matsubara Y, Hamada H, Nakagawa I, Uesugi F, Kubo T, Hiramatsu T. The effects of normothermic cardiopulmonary bypass on plasma concentrations of propofol in arterial, internal jugular bulb, and mixed venous blood. Masui 2003; 52: 716-21.

6. Hamada H, Nakagawa I, Uesugi F, Sakai A, Kimura M, Yasuuji M. Blood concentration of propofol during cardiopulmonary bypasscomparison between arterial and internal jugular venous blood. Masui 2001; 50: 724-30.

7. Myburgh JA, Upton RN, Grant C, Martinez A. Epinephrine, norepinephrine and dopamine infusions decrease propofol concentrations during continuous propofol infusion in an ovine model. Intensive Care Med 2001; 27: 276-82.

8. Adachi YU, Watanabe K, Higuchi H, Satoh T. The determinants of propofol induction of anesthesia dose. Anesth Analg 2001; 92: 65661.

9. Honan D, Doherty D, Frizelle H. A comparison of the effects on Bispectral index of mild vs. moderate hypothermia during cardiopulmonary bypass. Eur J Anaesthesiol 2006; 23: 385-90. 